\title{
Nonlinear Transmembrane Current Response of Micro Elelectroporation for Human Cancer Cells
}

\author{
Huiqi $\mathrm{He}^{\mathrm{a}}$, Donald C. Chang ${ }^{\mathrm{c}}$ and Yi-Kuen Lee ${ }^{\mathrm{a}, \mathrm{b} *}$ \\ ${ }^{a}$ Bioengineering Graduate Program, ${ }^{b}$ Dept of Mechanical Engineering, ${ }^{c}$ Dept of Biology \\ The Hong Kong University of Science and Technology, Hong Kong
}

\begin{abstract}
This paper presents a method to quantify the nonlinear electric current responses of electroporation (EP) on microchips for human cancer cells. Micro EP chips with three dimensional gold electrodes were fabricated with a modified electroplating process. Electrochemical impedance spectroscopy (EIS) was carried out with an electrochemistry analyzer on micro EP chips and a nonlinear equivalent circuit model was proposed to describe the dynamic response of the whole system. Using such a method, micro EP current was isolated from undesired leakage current to study the corresponding nano electropore dynamics under different input voltages. In addition, the EP efficiency results from electric measurements were confirmed by studies using fluorescence microscopy.
\end{abstract}

Keywords- electroporation, transmembrane current, HeLa cell, electrochemical impedance spectroscopic (EIS), equivalent circuit.

\section{INTRODUCTION}

Electroporation (EP) is a widely-used technique for delivery of many impermeable molecules such as drugs, oligonucleotides and plasmids into living cells [1]. The intact cell membrane represents a barrier to the transport watersoluble molecules due to the hydrophobic nature of the inner part of the lipid bilayer. When a strong electric field is applied to a cell, a nonuniform potential across the cell membrane is induced to a certain threshold and the cell membrane becomes porous, thus enabling entrance of various molecules that normally cannot easily cross the cell membrane [2-4]. The permeability of the cell membrane can be easily quantified by fluorescence microscopy [5]. The number of molecules of different sizes taken up by cells has also been studied under various electric pulse amplitudes and durations [6, 7]. At the same time, the measurements of the cellular electrical properties change during electroporation, such as transmembrane voltage $U_{m}(t)$, or transmembrane current $I_{m}(t)$, provide another way to describe cell membrane's permeability [8].

The current response on micro EP chips usually consists of electroporation, leakage (currents bypassing cells) and ionchannel currents as illustrated in Fig. 1. The application of an external electric field to the cell results in a considerable increase in the membrane permeability and conductance. The
EP current is directly related to the electric field induced nanoscale pore on the cell membrane, ranging from $1 \mathrm{~nm}$ to more than $100 \mathrm{~nm}$ [9]. In order to have a deeper understanding about EP, the EP current needs to be isolated from the other currents. Usually, the conductivity change of the artificial lipid bilayer or cell membrane were detected by connecting the membrane with a pipette or patch clamp $[10,11]$ to ensure a tight seal. In this case, the leakage current could be neglected. However, the manipulation of the cells using a pipette causes difficulties in operation. To date, there is no comprehensive work to investigate the EP current response, which is related to the nano electropore dynamics, from the bulk cell suspension experiments.

Electrochemical method has been widely adopted in biological applications. Electrical cell-substrate impedance sensor (ECIS) adopted electrochemical impedance spectroscopic (EIS) method, which can provide the information of an electrode/electrolyte interface, to monitor the concentration, growth and physiological states of cells cultured on electrodes [12]. EIS measurements can also be used to build an equivalent circuit to describe the electric characteristics of the system.

In this paper, the experimental impedance measurements were first carried out. Then, theoretical modeling and analysis were applied to obtain a nonlinear equivalent circuit model to describe the whole system. This model was able to predict the micro EP current which related to the corresponding electropore dynamics.

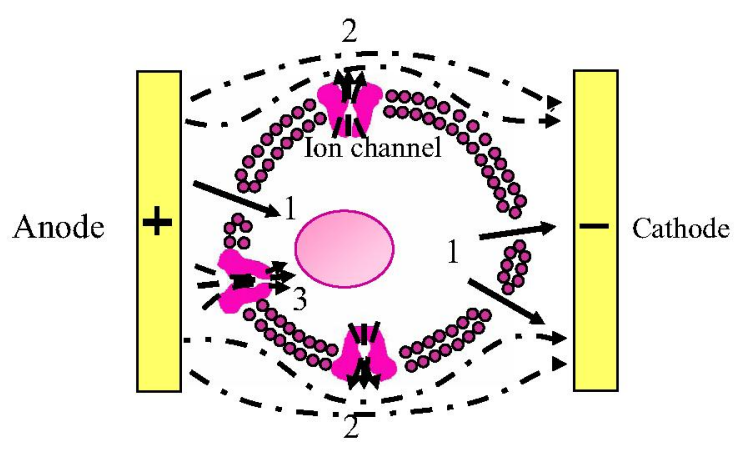

Fig. 1. Schematic diagram of three electric currents across an electroporated cell. 1.EP current (solid line), 2. leakage current (dash dot), 3. ion channel current (dash line). 


\section{DEVICE FABRICATION}

Micro EP chips with three-dimensional gold micro electrodes were fabricated by using a modified electroplating process. A gold seed layer was patterned first using the lift-off technique, so that no additional step was required to etch the gold layer in an iodide bath after the electroplating process. This can prevent the electroplated structure from being attacked, and ensures that it is consistent with the original design. The detailed fabrication process was described elsewhere [7]. A photo of a packaged micro EP chip and the SEM close-up view of the fabricated 3D electrode structures are shown in Fig. 2.

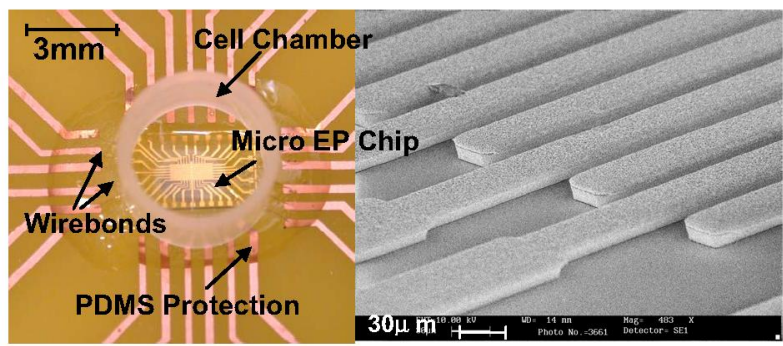

Fig. 2. A photo of a packaged micro EP chip (left) and the SEM close-up view of the $3 \mathrm{D}$ microelectrodes (right).

\section{EXPERIMENTAL RESULTS}

Fig. 3 shows the cyclic voltammetry of 3D electrodes at a scan rate of $100 \mathrm{mV} / \mathrm{s}$ in a commonly used poration medium $(\mathrm{pH}=7.4)$ using Autolab PGSTAT 30 Potentiostat/Gal-vanostat system (Eco Chemie). Cyclic voltammetry provides the information of the kinetic barrier of a bare gold electrode and its surrounding electrolyte interface. The standard potential of hydrolysis on the microchip was determined to be around $1.5 \mathrm{~V}$.

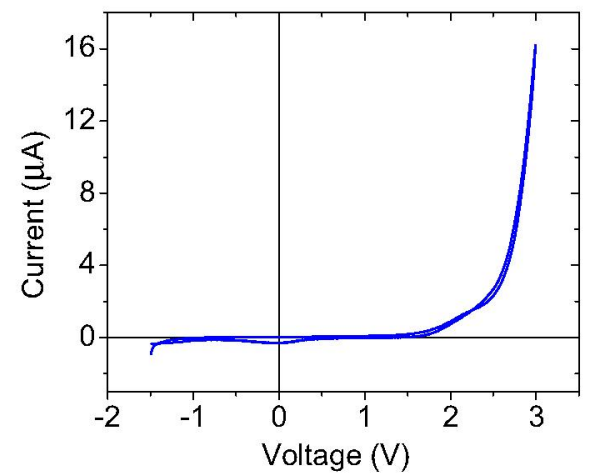

Fig. 3. Cyclic Voltammogram of a bare gold electrode in poration medium.

In the experiment, $15 \mu \mathrm{L}$ poration medium and human cervical cancer cell $\left(\mathrm{HeLa}\right.$ cell) suspension $\left(10^{8}\right.$ cells $\left./ \mathrm{mL}\right)$ were loaded onto the micro EP chip respectively before electric pulse treatment. The purpose of using high concentration cell suspension was to block the space between the two electrodes and reduce the current bypassing the cell. Current responses were recorded using PCI 6110 DAQ card
(National Instrument, TX, USA) for a sequence of $0.2 \mathrm{msec}$ pulses with increasing pulse amplitude ranging from $1 \mathrm{~V}$ to 6 V. As shown in Fig. 4, it is obvious the current measured in the poration medium is larger than the current in the cell suspension, since the cells act as an insulator in the intact state to block the current passing across the two electrodes. At an applied voltage larger than $3 \mathrm{~V}$, it can be seen there is a current "jump" in cell suspension. The current jump is due to the increase of cell membrane conductance induced by the electric pore generation. Thus, the dynamics of membrane conductance changes can be characterized using transmembrane current data.

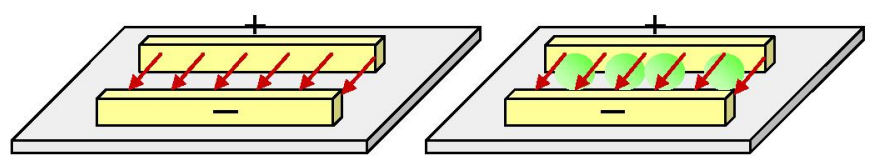

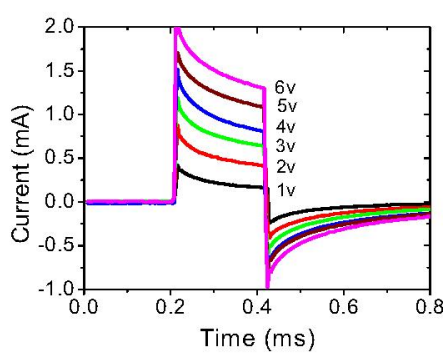

(a)

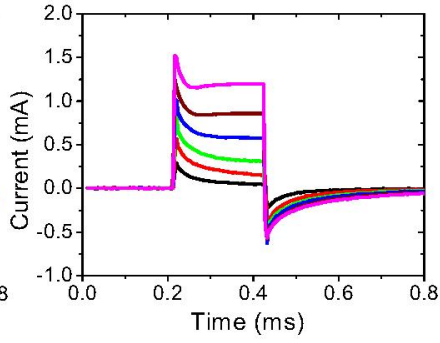

(b)
Fig. 4 . Current responses to different input electric pulses ranging from $1 \mathrm{~V}$ to $6 \mathrm{~V}$ for (a) Current response of the poration medium, (b) HeLa cell suspension.

\section{DATA ANALYSIS}

In order to isolate the EP current, the non-linear leakage current bypassing the cells should be estimated and subtracted from the total electric current. Electrochemical impedance spectroscopy (EIS) was carried out with Autolab PGSTAT 30 at a range of $10 \mathrm{~Hz}$ to $100 \mathrm{kHz}$ (Fig. 5(a)). According to the Nyquist plot of impedance (open circle in Fig.5(c) and (d)), a modified Randle's equivalent circuit [13] shown in Fig. 5(b) was found to fit the impedance data adequately over the working frequency range.

Therefore, the non-linear leakage current containing both nonfaradaic and faradaic (at a high voltage input) components can be described solely by an equivalent circuit shown in Fig. 5 (b). The equivalent circuit contains four components which are defined by five parameters, in which $R_{\mathrm{ct}}$ is a charge transfer resistance and CPE is a Constant Phase Element. $Z_{\text {war }}$ represents the Warburg impedance associated with diffusive ion transportion and $R_{s}$ is the resistance of electrolyte between the two electrodes [13]. Fig. 5 (c) and (d) shows the Nyquist impedance plot detected in the experiment (open circuit) and fitted by the equivalent circuit (solid line) for both poration medium and cell suspension. Five parameters, $R_{s}, Y_{0}, n, R_{c t}$ and $W_{0}$ for both PM and cell suspension at zero potential are listed in Table 1. 
TABLE I

Predicted parameters for the equivalent circuit (Fig. 5(b)).

\begin{tabular}{|c|c|c|}
\hline Component & PM & Cell Suspension \\
\hline$R_{s}$ & $2.685 \mathrm{k} \Omega$ & $4.4 \mathrm{k} \Omega$ \\
\hline$Q_{1} Y_{0}$ & $1.345 \mathrm{e}-11$ & $2.178 \mathrm{e}-11$ \\
\hline$Q_{1} n$ & 0.6378 & 0.9449 \\
\hline$R_{c t}$ & $32.9 \mathrm{k} \Omega$ & $1.578 \mathrm{M} \Omega$ \\
\hline$W_{0}$ & $4.903 \mathrm{e}-8$ & $1.759 \mathrm{e}-7$ \\
\hline
\end{tabular}

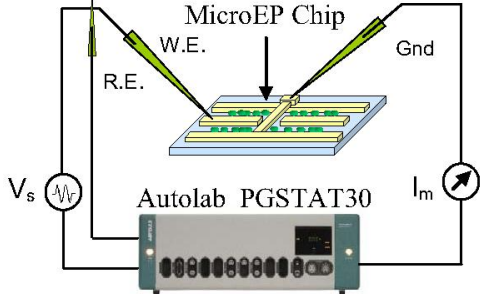

(a)

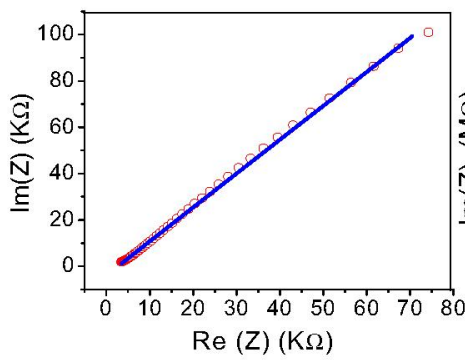

(c)

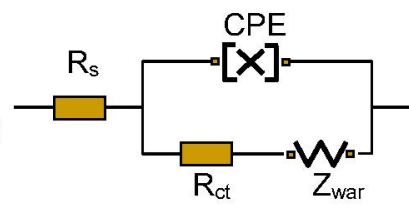

(b)

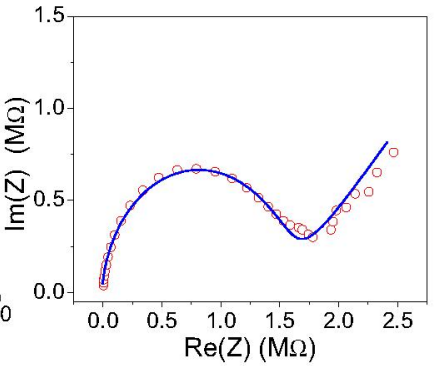

(d)
Fig. 5. (a) Electrochemical impedance spectroscopy setup, (b) Equivalent circuit used to model the impedance data. Fitted (solid line) and experimental (open circle) Nyquist plots of impedance spectra in poration medium (c) and cell suspension (d) (frequency from $10 \mathrm{~Hz}$ to $100 \mathrm{kHz}$ ).

According to the equivalent circuit, the impedance of whole system, $Z$, can be calculated as follows:

$$
Z(j \omega)=R_{s}+\frac{1+R_{c t} W_{0}(j \omega)^{0.5}}{R_{c t} Y_{0}^{n}(j \omega)^{n} W_{0}(j \omega)^{0.5}+W_{0}(j \omega)^{0.5}+\left(Y_{0}\right)^{n}(j \omega)^{n}}
$$

where $Z_{\mathrm{CPE}} \equiv 1 /\left(Y_{0} j \omega\right)^{n}, \quad Z_{\mathrm{war}} \equiv 1 / W_{0}(j \omega)^{0.5}, \quad 0<n<1, \omega=2 \pi f$ with $f$ being the frequency. The current response, $I(s)$, to an input electric pulse with the amplitude of $V$ and duration of $t_{2^{-}}$ $t_{1}$ can be derived as follows:

$$
\begin{aligned}
& I(s)=\frac{U(s)}{Z(s)}=\frac{V\left(e^{-s t_{1}}-e^{-s t_{2}}\right)}{s \times Z(s)} \\
& =\frac{V \times\left(e^{-s t_{1}}-e^{-s t_{2}}\right)\left(R_{c t} W_{0} Y_{0}^{n} s^{n+0.5}+Y_{0}^{n} s^{n}+W_{0} s^{0.5}\right)}{s\left(R_{s} R_{c t} W_{0} Y_{0}^{n} s^{n+0.5}+R_{s} Y_{0}^{n} s^{n}+\left(R_{s}+R_{c t}\right) W_{0} s^{0.5}+1\right)}
\end{aligned}
$$

An inverse Laplace transform (ILT) of Eqn. (2) would lead to a current response in the time domain, $I(t)$. However, since $n$ is not an integer, it is not possible to convert Eqn. (2) to time domain using simple ILT analysis. Here we introduce a numerical inverse Laplace transform - the Weeks method [14]. The Weeks method is based on a Laguerre expansion and bilinear transformations, and it can be implemented with the
FFT. The numerical ILT program using Weeks method was developed in MATLAB by J.A.C. Weideman [15]. With the ILT program, the current response, $I(t)$, cab be easily calculated. As shown in Fig. 6, the numerical current response obtained from the ILT of Eqn. 2 is quite consistent with the measured data, with a standard deviation of 0.0715 $\left(R^{2}=0.9735\right)$, which verified the accuracy of equivalent circuit model and the ILT program.

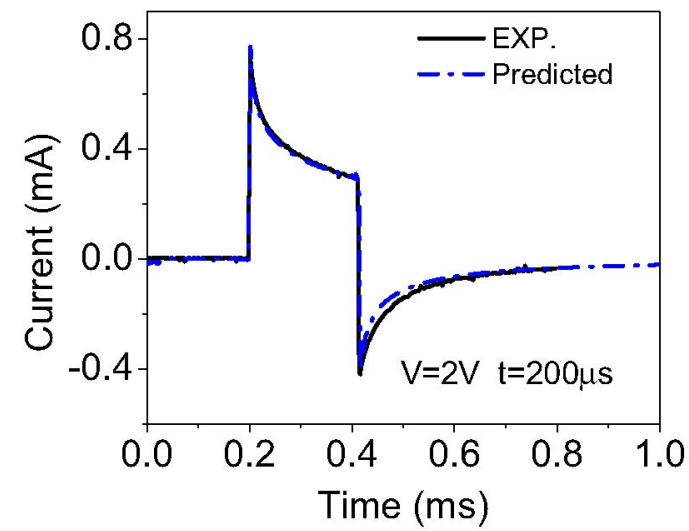

Fig. 6. Measured current response of the poration medium (black solid line) and predicted current response from Eqn. 2 (bule dash dot).

The five-parameter equivalent circuit under different applied voltage could be used to predict the non-linear leakage current. Based on the parameters determined from the EIS measurement, a short pulse (e.g. $50 \mu \mathrm{s}$ ) which will not induce electroporation (checked by microscopy) was also utilized for adjusting the parameters especially in the case of high applied voltage. This is because EIS measurement usually takes minutes to scan the whole frequency while at a high potential, hydrolysis has already occurred and it will affect the accuracy of the circuit model.

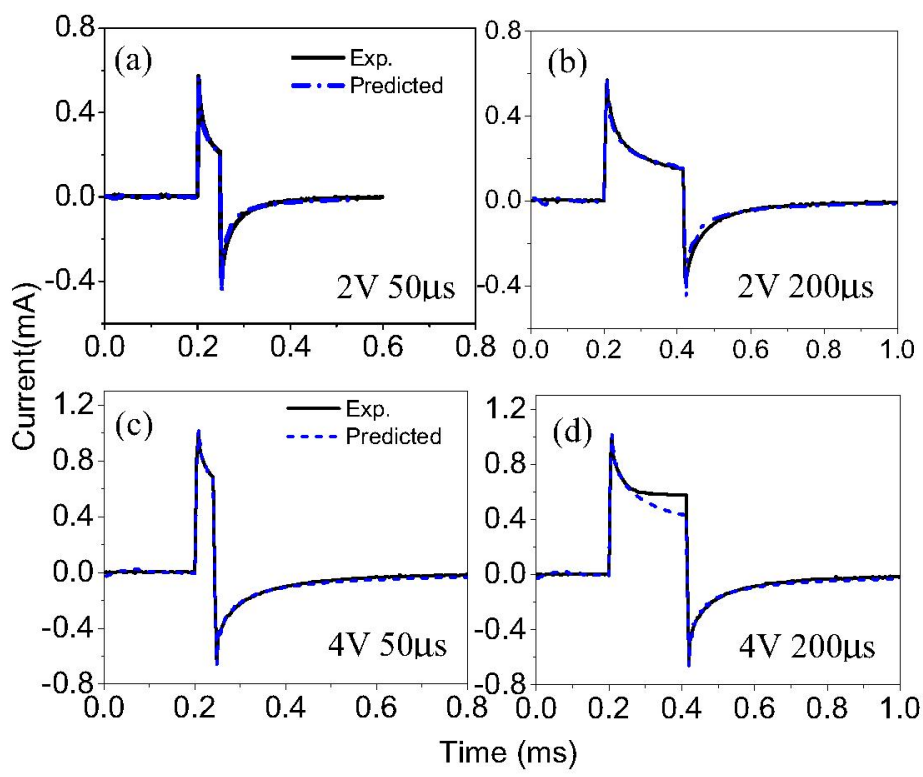

Fig. 7 Current response of measured data (black solid line) and predicted leakage current through the cell suspension under $2 \mathrm{~V}$ (blue dash dot) or $4 \mathrm{~V}$ (blue dash line). 
Fig. 7 shows the method to predict current response in cell suspension according to the circuit model determined by a short pulse. Firstly, five parameters in the equivalent circuit were determined according to the measured data under a short pulse by Least-Square curve fitting (Figs. 7(a) and (c)). Then the equivalent model was used to predict the leakage current for a longer pulse, like $200 \mu \mathrm{s}$. It is obvious, for small pulse amplitude, the measured data is quite consistent with the predicted one as in Fig. 7(b). While for a large amplitude pulse, the difference between the measured data and the predicted one is detectable, indicating the increased transmembrane current due to electric-induced pores on the cell membrane. Moreover, with the increase of the pulse amplitude, the difference becomes larger as shown in Fig. 8.

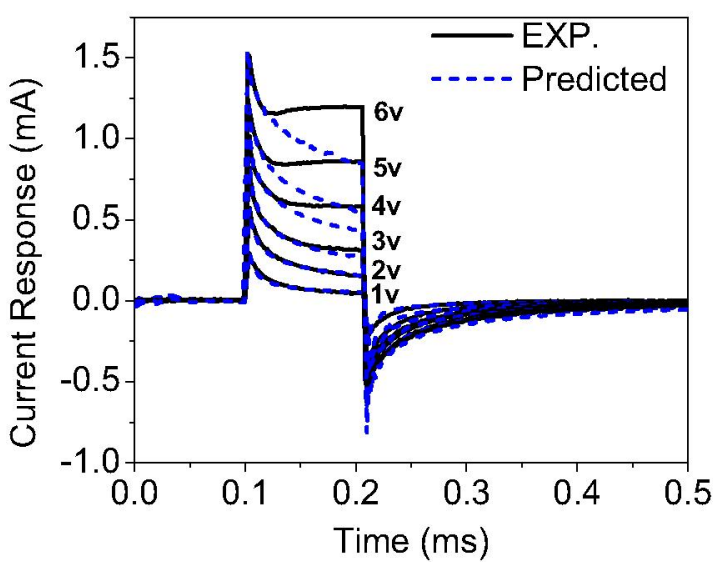

Fig. 8. Measured current response of cell suspension (solid line) and predicted current response from Eqn. 2 (dash line).

The net transmembrane currents were then isolated by subtracting the leakage current from the measured current response of the cell suspension. As shown in Fig. 9, the transmembrane currents under different electric pulses (amplitude from $1 \mathrm{~V}$ to $6 \mathrm{~V}$ and the pulses duration of $0.2 \mathrm{~ms}$, $0.8 \mathrm{~ms}$ and $2 \mathrm{~ms}$ ) were recorded. Similar to the result previously obtained from fluorescence microscopy with Propidium Iodide(PI) dye indicator [7], the EP current can only be detected when external applied voltage is larger than some critical value, e.g. $3 \mathrm{~V}$ in $0.2 \mathrm{~ms}$ and $2 \mathrm{~V}$ in $0.8 \mathrm{~ms}$ and $2 \mathrm{~ms}$. The threshold voltage indicates the minimum voltage to induce the pores on the cell membrane under different pulse duration. And the longer the pulse duration, the lower the pulse amplitude required for a detectable EP current. The two methods are in good agreement at the threshold voltage induce cell membrane permeability, as shown in Fig. 10.

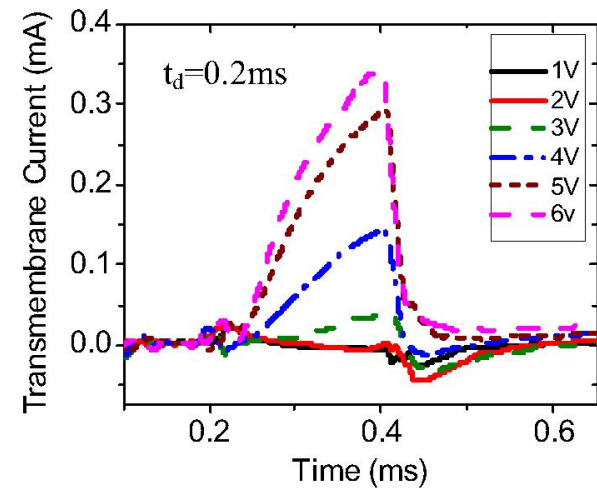

(a)

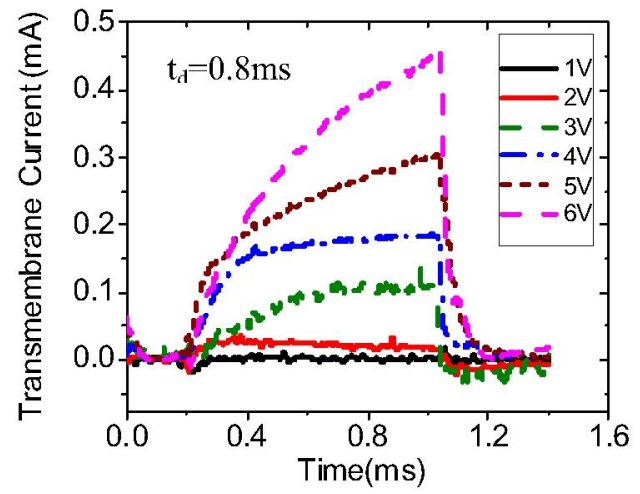

(b)

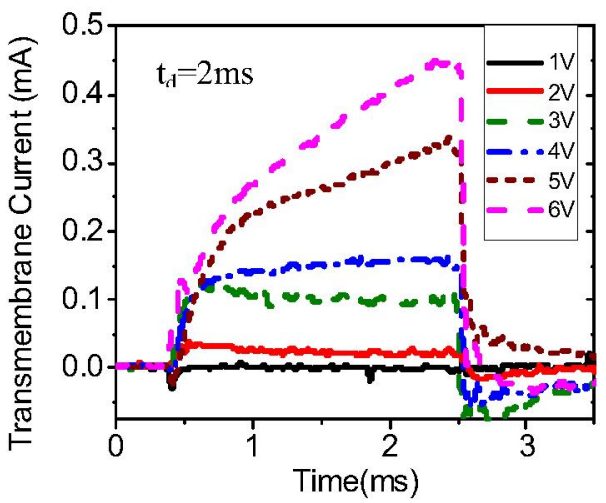

(c)

Fig. 9. Isolated net transient transmembrane current under pulse amplitude of $1-6 \mathrm{~V}$ and pulse duration (a) $0.2 \mathrm{~ms}$ (b) $0.8 \mathrm{~ms}$ and (c) $2 \mathrm{~ms}$.

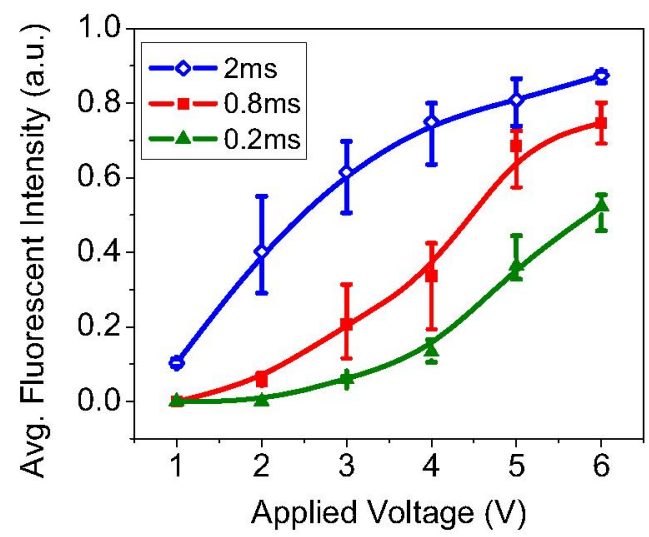

Fig. 10. Average fluorescence intensity as a function of applied voltage with duration of $0.2 \mathrm{~ms}, 0.8 \mathrm{~ms}$ and $2 \mathrm{~ms}$. Each data point from the average of 10 single HeLa cells. 


\section{CONCLUSION}

Micro electroporation chips with 3D micro electrodes have been designed and fabricated, using MEMS technology. A non-linear equivalent circuit, with the help of impedance measurement, was proposed for micro electroporation. Undesired leakage currents bypassing the cells on the chip were predicted by the equivalent circuit modeling and simulation. The increase of transmembrane current due to electroporation has been successfully determined under different input voltages and pulse durations. This provides an alternative method to quantify the temporal changes in cell membrane conductance and electropores in response to electroporation. The EP results from our proposed electric method are in good agreement with the results from fluorescence microscopy.

\section{ACKNOWLEDGEMENTS}

This work is supported by Hong Kong Research Grants Council (Project Ref No. 616205).

\section{REFERENCES}

[1] D. C. Chang, B. M. Chassy, J. A. Saunders, A. E. Sowers, "Guide to Electroporation and Electrofusion," Academic Press, San Diego, 1992, pp. 1-6.

[2] E. Neumann, M. Schaefer-Ridder and M, Y. Wang, "Gene Transfer into mouse lyoma cells by electroporation in high electric fields," $E M B O J$. vol. 1, pp. 541-845, 1982.

[3] U.Zimmermann, "Electric field-mediated fusion and related electrical phenomena", Biochim. Biophy. Acta.Vol.694, pp. 227-277, Nov. 1982.

[4] J. C. Weaver and Y. A. Chizmadzhev, "Theory of electroporation: A review," Bioelectrochemisry and Bioenergetics, vol.41, pp.135-190, Dec. 1996.

[5] D.C.Bartoletti, G.I.Harrison and J.C.Weaver, "The number of molecules taken up by electroporated cells: quantitative determination," FEBS Lett. vol.256, pp. 4-10, Oct. 1989.
[6] H.Liang, W.J.Purucker, D.A.Stenger, R.T.Kubiniec and S.W.Hui, "Uptake of fluorescence-labeled dextrans by $10 \mathrm{~T}$ $1 / 2$ fibroblasts following permeation by rectangular and exponential-decay electric field pulse". Biotechniques, vol. 6, pp. 550-558, Jun. 1988.

[7] H.He, D.C.Chang and Y.-K. Lee, "Using a micro electroporation chip to determine the optimal physical parameters in the uptake of biomolecules in HeLa cells," Bioelectrochemistry, vol.71, pp. 80-85, 2006.

[8] I. G. Abidor, V. B. Arakelyan, L.V. Chernomordik, Yu. A. Chizmadzhev, V. E. Pastushenko and M. R. Tarasevich, "Electric breakdown of bilayer lipid membranes I. The main experimental facts and their qualitative discussion," Bioelectrochemistry and bioenergetics, vol. 6, pp. 37-52, Mar.1979.

[9] D. C. Chang and T. S. Reese, "Changes in membrane sturcture induced by electroporation as revealed by rapidfreezing electron microscopy," Biophysical Journal, vol. 58, pp. 1-12, 1990.

[10]F.Ryttsen, C.Farre, C. Brennan, S.G.Weber, K.Nolkrantz, K.Jardemark, D.T.Chiu, and O. Orwar, "Characterization of single-cell electroporation by using patch-clamp and fluorescence microscopy," Biophysical Journal, vol. 79, pp1993-2001, october, 2000.

[11]T.R.Gowrishankar, W.Chen and R.C.Lee, "Non-linear microscale alterations in membrane transport by electropermeabilization," Ann. N. Y. Acad. Sci. vol. 858, pp205-216, 1998.

[12]I. Giaever, C. R. Keese, "Monitoring fibrolblasts behavior in tissue culture with an applied electric field," Proc. Natl. Acad. Sci. vol.81, pp.3761-3764, Jun.1984.

[13]E. Barsoukov, et al., Impedance Spectroscopy: Theory, Experiment, and Applications, Wiley-Interscience, Hoboken, N.J, 2005.

[14]B. S. Garbow, G. Giunta, J. N. Lyness, and A. Murli, "Software for an implementation of Weeks' method for the inverse of the Laplace Transform," ACM TOMS, Vol. 14, pp.163-170, June, 1988.

[15]J .A. C. Weideman, SIAM J. Sci. Comput., vol. 21, pp. 111-128, 1999. 